\title{
Tomographic Reconstruction in Atom Probe Microscopy: Past, Present... Future?
}

\author{
B. Gault, ${ }^{* * *}$ M. P. Moody, ${ }^{* *}$ E. A. Marquis, ${ }^{*}$ F. de Geuser, ${ }^{* * *}$ B. P. Geiser, $* * * *$ D. J. Larson, \\ $* * * *$ T. F. Kelly****, S.P. Ringer, ${ }^{* *}$ George D.W. Smith* \\ * Department of Materials, University of Oxford, Parks Road, Oxford OX1 3PH, UK. \\ ** Australian Key Centre for Microscopy and Microanalysis, Madsen Building F09, The University \\ of Sydney, NSW 2006, Australia. \\ *** Laboratoire Science et Ingénierie des MAtériaux et Procédés, UMR CNRS 5266, INP Grenoble - \\ BP 46. Domaine Universitaire - 101 rue de la Physique 38402 Saint Martin d'Hères, France. \\ **** Imago Scientific Instruments Corporation, 5500 Nobel Drive, Madison, WI 53711-4951 USA
}

Atom probe microscopy enables the three-dimensional characterization of materials, combining high spatial and elemental resolution [1]. Due largely to the recent renaissance of the pulsed-laser atom probe and the development of focused-ion beam specimen preparation, the technique is currently experiencing a period of significant growth worldwide [2]. Whereas most aspects of the technique have evolved momentously throughout the last decade, the procedure used to build a tomographic image of the analyzed volume has for the most part remained unchanged. The lateral coordinates are estimated based on a simple projection model. The in-depth coordinate of each detected atom is sequentially calculated on the assumption that each contributes to the depth by an increment proportional to its atomic volume. A corrective term is consequently applied to account for the original curvature of the specimen surface.

This somewhat basic procedure introduced by Bas et al. in 1995 [3], after the work by Blavette et al. in 1982 [4], still produces impressive results. Atomic planes are commonly visible in the tomographic reconstruction relative to the atom probe analysis of crystalline materials, especially when the direction normal to the atomic planes is close to the direction of analysis. Various treatments applied to the data, especially Fourier transform [5,6] or so-called spatial distribution maps [7], enable further characterization of these crystallographic features. However, the exact performance of this reconstruction procedure has never been accurately assessed.

In the present work, the current reconstruction procedure will be reviewed. Various improvements linked to the development of wide-angle atom probe will be detailed relative to the development of a calibration methodology for the reconstruction procedure [8]. Further, methods to study the performance of the technique in terms of spatial resolution will be detailed, based on the use of advanced spatial distribution maps [9]. Finally, potential new methods to build tomographic reconstructions, based on the use of field desorption micrographs, will be discussed.

[1] D. Blavette et al. Rev. Sci. Instr. 64 (1993) 2911-2918.

[2] A. Cerezo et al., Microsc. Microanal. 13, (2007) 408-417.

[3] P. Bas et al., Appl. Surf. Sci. 87/88 (1995) 298-304.

[4] D. Blavette et al., Revue Phys. Appl. 17 (1982) 435-440.

[5] P.J. Warren, et al., Microsc. Microanal. 4 Suppl.2 (1998) 86-87.

[6] F. Vurpillot et al., J. Microsc. 203, pt. 3 (2001) 295-302.

[7] B. Geiser et al., Microsc. Microanal. 13, (2007) 437-447.

[8] B. Gault et al. Microsc. Microanal. 14, (2008) 296-305.

[9] M.P. Moody et al., accepted in Ultramicroscopy (Jan. 2009). 

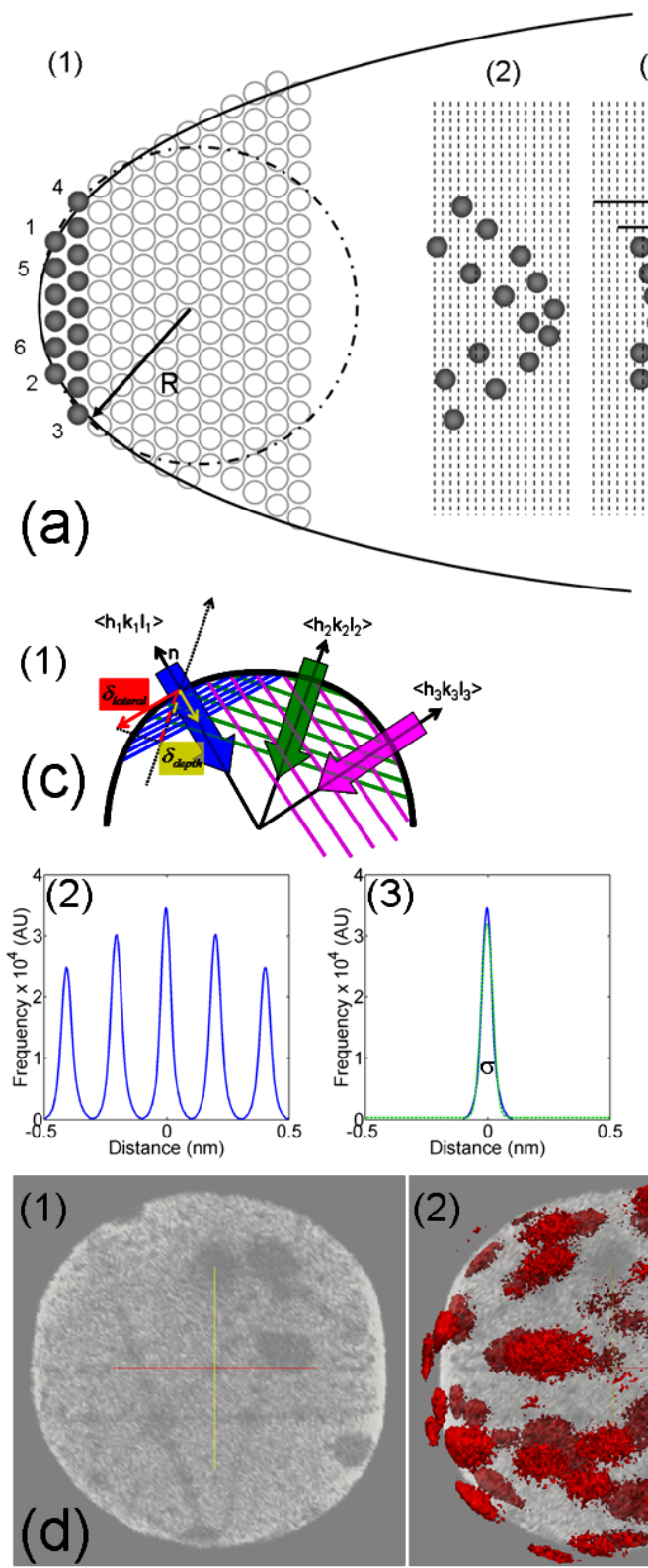

(3)
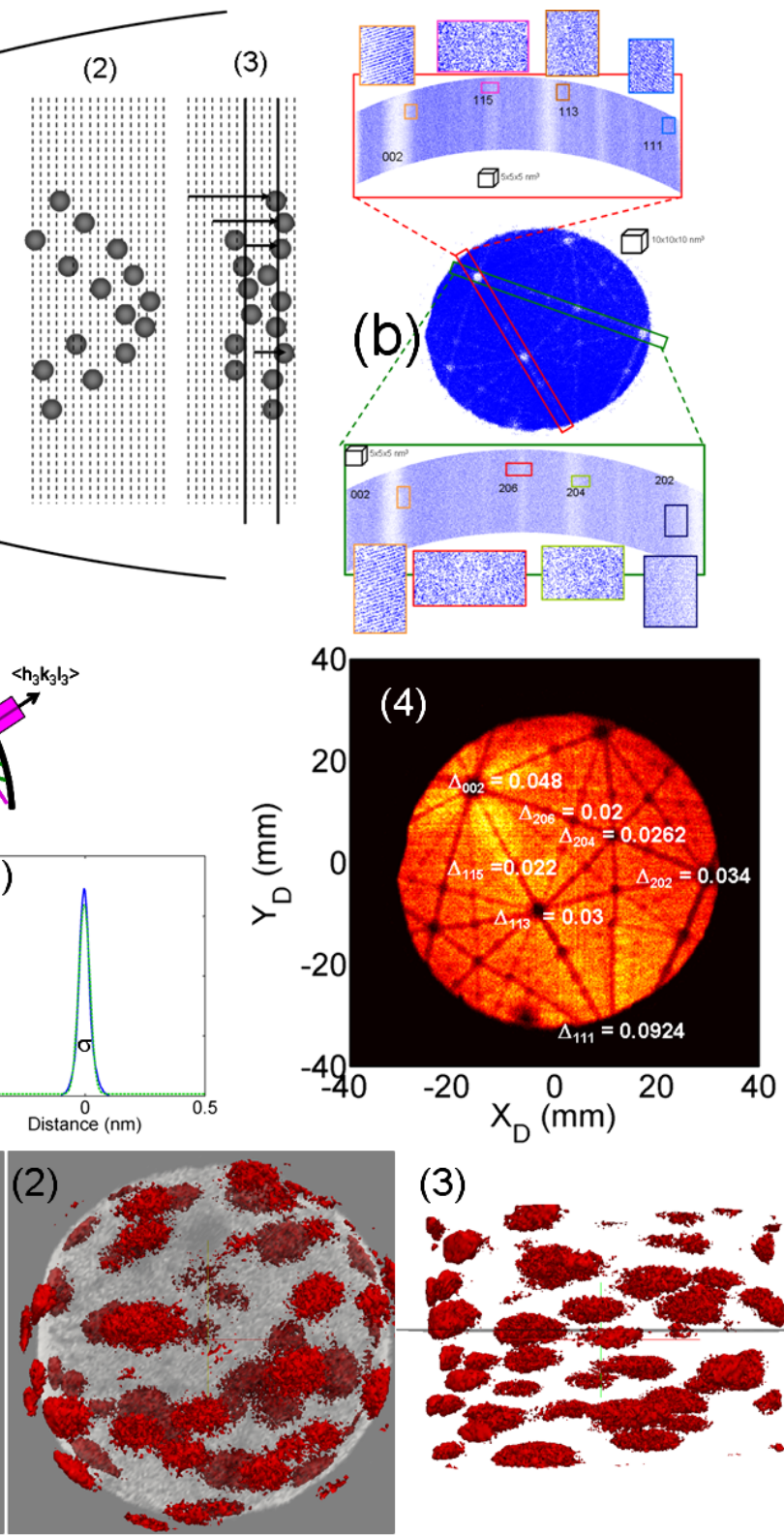

(3)

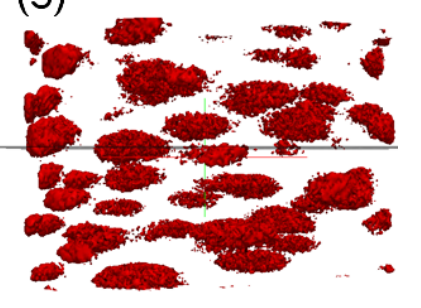

Figure 1: (a) Schematic overview of the reconstruction procedure: the order in which the atoms are evaporated (1) is used to progressively build the in-depth dimension by attributing an increment (2). A correction term is consequently applied to account for the curvature. (b) Pure aluminum analysis showing evidence for several atomic plane families. (c) Procedure used to estimate the spatial resolution for several different atomic plane families (1) using a z-SDM (2), the central peak of which is fitted with a Gaussian function (3), highlighting the variations of the spatial resolution over the surface of the specimen (4). (d) Reconstruction of an AlAg alloy based on the computation of chemically resolved desorption map: the $\mathrm{Al}$ is displayed as a slice (1), which shows the characteristic pole structure, Ag precipitates are readily observable in 3D (2-3). This new reconstruction procedure is proposed to be used correlatively with electron tomography. 